\title{
Análisis isotópico de camélidos de sitios arqueológicos del Holoceno tardío en el norte de la quebrada de Humahuaca (Argentina)
}

\author{
Anahí Hernández y Luciano O. Valenzuela \\ Recibido 20 de febrero 2021. Aceptado 15 de julio 2021
}

\begin{abstract}
RESUMEN
En este trabajo se exponen los primeros resultados isotópicos de restos óseos de camélidos de dos sitios arqueológicos del norte de la quebrada de Humahuaca (Jujuy, Argentina): Antumpa (1900-1300 AP; 3300 msnm) y Cóndor II (1100-960 AP; 3810 msnm). El objetivo es generar información sobre la explotación y manejo de este recurso en una región y para un lapso temporal que cuentan con escasos datos isotópicos. Se presentan 11 pares de valores de $\delta^{13} \mathrm{C}$ y $\delta^{15} \mathrm{~N}$ medidos sobre colágeno óseo de camélidos arqueológicos que aportan nueva información para los estudios isotópicos regionales. Los valores de $\delta^{13} \mathrm{C}$ obtenidos oscilan entre $-18,3 \%$ y $-14,0 \%$ (media $=-16,2 \%$ ); y los de $\delta^{15} \mathrm{~N}$, entre $6,4 \%$ y $8,9 \%$ (media $=$ $7,7 \%$ ). Se registraron diferencias en los valores de $\delta^{13} \mathrm{C}$ de camélidos de Antumpa y Cóndor II y ausencia de variaciones en las mediciones de $\delta^{15} \mathrm{~N}$ que podrían explicarse por la distinta altitud entre los sitios estudiados, aunque no se descarta el factor temporal como fuente de variabilidad isotópica alternativa. Además, se registró una muy baja variación de los valores de $\delta^{13} \mathrm{C}$ y $\delta^{15} \mathrm{~N}$ entre las especies de camélidos representadas, que deberá corroborarse en futuras investigaciones con la incorporación de nuevas muestras.
\end{abstract}

Palabras clave: Isótopos estables; Camélidos; Zooarqueología; Noroeste argentino.

\section{Isotopic analysis of camelids from late Holocene archaeological sites in the north of Quebrada de Humahuaca (Argentina)}

\begin{abstract}
In this paper, the first isotopic results on camelid bone from two archaeological sites located in the north of quebrada de Humahuaca (Jujuy, Argentina) are presented: Antumpa (1900-1300 BP; 3300 masl) and Cóndor II (1100-960 BP; 3810 masl). The aim of this work is to generate information about the exploitation and management of camelids in a region and for a time period with little isotopic data. We present $\delta^{13} \mathrm{C}$ and $\delta^{15} \mathrm{~N}$ values measured on bone collagen of eleven archaeological camelids, which provide new and unique information for isotopic studies in this region. $\delta^{13} \mathrm{C}$ value ranges between $-18.3 \%$ and $-14.0 \%$ o (mean $=-16.2 \%$ ) and $\delta^{15} \mathrm{~N}$ values ranges between $6.4 \%$ and $8.9 \%$ (mean $=7.7 \%$ ). Differences in $\delta^{13} \mathrm{C}$ values and no variations in $\delta^{15} \mathrm{~N}$ values between Antumpa and Cóndor II were recorded. This can be explained by differences in elevation between the sites. However, the temporal factor is not ruled out as a source of alternative isotopic variability. Furthermore, we observe very low variations of $\delta^{13} \mathrm{C}$ and $\delta^{15} \mathrm{~N}$ values among the species of camelids, which should be corroborated in future research through the incorporation of new samples.
\end{abstract}

Keywords: Stable isotopes; Camelids; Zooarchaeology; Argentina northwest.

Anahí Hernández. Consejo Nacional de Investigaciones Científicas y Técnicas (CONICET). Departamento de Arqueología, Facultad de Humanidades y Artes, Universidad Nacional de Rosario. Entre Ríos 758 (S2000CRN), Rosario, Santa Fe, Argentina. E-mail: anahihernandez87@hotmail.com

Luciano Valenzuela. CONICET. Laboratorio de Ecología Evolutiva Humana, Facultad de Ciencias Sociales, Universidad Nacional del Centro de la Provincia de Buenos Aires (UNCPBA). Calle 508 N 88I (B763IXAF), Quequén, Buenos Aires, Argentina. E-mail: lucianoovalenzuela@gmail.com

Intersecciones en Antropología 22(2), julio-diciembre: 183-194. 2021. ISSN-e 1850-373X

https://doi.org/10.37176/iea.22.2.2021.624

Facultad de Ciencias Sociales - UNICEN - Argentina 


\section{INTRODUCCIÓN}

Los camélidos sudamericanos fueron un recurso económico fundamental en el área andina y tuvieron un rol central en la subsistencia de las poblaciones humanas prehispánicas del Noroeste argentino (NOA) (Mengoni Goñalons y Yacobaccio, 2006). El predominio de sus restos óseos en los sitios arqueológicos del área y su representación en el arte rupestre regional demuestran este hecho (Hernández Llosas, 2001; Olivera, 2001; Gallardo y Yacobaccio, 2007).

La vicuña (Vicugna vicugna) y el guanaco (Lama guanicoe) constituyen los taxones silvestres que componen la Familia Camelidae en América, mientras que la llama (Lama glama) y la alpaca (Vicugna pacos) son los taxones domésticos. A excepción de esta última especie, adaptada a ambientes húmedos de altura no frecuentes en el NOA, los especímenes óseos de las tres restantes están entremezclados en el registro arqueológico de la región desde comienzos del Holoceno tardío (Yacobaccio, 2001). La explotación faunística implicó la combinación de estrategias de caza y pastoreo (Olivera y Palma, 1997) y diferentes tipos de interacciones entre las poblaciones humanas y de camélidos.

Las prácticas pastoriles andinas presentan particularidades que las diferencian del pastoreo desarrollado en otras partes del mundo. Los estudios etnográficos señalan, entre sus características, el nivel de asociación con las prácticas agrícolas y la movilidad estacional de los rebaños en busca de pasturas y fuentes de agua en diferentes escalones altitudinales (Bonavia, 2008; Yacobaccio, 2014). Estas particularidades habrían incidido en la alimentación de los rebaños como resultado de su pastoreo en distintas comunidades vegetales o por la intervención humana en la dieta de los rebaños de llamas mediante, por ejemplo, el uso del rastrojo de los cultivos como fuente suplementaria de las pasturas naturales (Yacobaccio et al., 2010; Samec, 2014; Grant y Olivera, 2016). Asimismo, las características fitogeográficas de los territorios de alimentación de los grupos de camélidos silvestres y su localización en diferentes altitudes, así como los hábitos territoriales de ambas especies, habrían influido en la dieta de estos animales (Puig et al., 1997; Borgnia et al., 2010).

Los análisis de isótopos estables constituyen una técnica analítica frecuentemente empleada para estudiar la dieta de poblaciones humanas y animales (Ambrose, 1993; Srur et al., 2012; Fuchs et al.,
2015; Kilian Galván et al., 2016; Kilian Galván, 2018). Estos estudios brindan información sobre temas de interés para la arqueología, y particularmente para la zooarqueología, como la movilidad pastoril (Balasse et al., 2002; Gerling et al., 2017), la diferenciación de especies con tamaño y morfología similar (Gorlova et al., 2015) y las estrategias de explotación y manejo de animales (Balasse et al., 2002; Finucane et al., 2006; Dufour et al., 2014; Zavodny et al., 2015). En el caso de los camélidos sudamericanos, son numerosos los trabajos que buscan comprender su variabilidad isotópica mediante el estudio de restos modernos y arqueológicos (Fernández y Panarello, 1999-2001; Finucane et al., 2006; Izeta et al., 2009; Yacobaccio et al., 2009, 2010; Thornton et al., 2011; Dantas, 2012; Szpak, 2013; Dufour et al., 2014; Samec et al., 2014, 2018; Samec, 2015; Grant y Olivera, 2016; Panarello y Mondini, 2016; López Mendoza et al., 2017; Grant et al., 2018; Mondini et al., 2019); entre los que se destaca, para la quebrada de Humahuaca, el estudio realizado por Mengoni Goñalons (2014). Sin embargo, prácticamente no se cuenta con datos isotópicos de camélidos arqueológicos del norte de dicha quebrada, y menos aún de contextos del Holoceno tardío, con excepción de las mediciones realizadas en especímenes del sitio Inca Cueva 4 pero que datan del Holoceno temprano y medio (Samec, 2015).

El objetivo principal de este trabajo es analizar la explotación y manejo de camélidos en las nacientes de la quebrada de Humahuaca entre ca. 2000-900 años AP mediante estudios isotópicos (carbono y nitrógeno) de colágeno óseo de especímenes de los sitios Antumpa y Cóndor II. Estos se localizan en distintos ambientes y altitudes, por lo cual se espera que los valores isotópicos de ambos conjuntos varíen en función de ello, de manera que se registraría un mayor aporte de especies $\mathrm{C}_{4}$ en la dieta de los camélidos procedentes de Antumpa. Las muestras estudiadas corresponden a diferentes lapsos temporales dentro del Holoceno tardío, lo que podría constituir otra fuente de variabilidad isotópica a ser considerada. Complementariamente, exploramos las composiciones isotópicas de las distintas especies de camélidos representadas.

Los resultados obtenidos son comparados con información generada por diversos investigadores/as a partir del estudio de especies vegetales y animales modernos de la Puna Seca de Jujuy y quebradas de transición a la puna (Fernández y Panarello, 19992001; Yacobaccio et al., 2009, 2010; Samec, 2011, 
2014, 2015; Samec et al., 2017, 2018). La presentación de estos once pares de valores de $\delta^{13} \mathrm{C}$ y $\delta^{15} \mathrm{~N}$ constituye un primer aporte al conocimiento de la composición isotópica de restos óseos de camélidos del Holoceno tardío para el área de estudio.

\section{Isótopos de carbono y nitrógeno, y su variabilidad}

Las plantas fijan el $\mathrm{CO}_{2}$ atmosférico mediante tres vías fotosintéticas, Calvin-Benson $\left(\mathrm{C}_{3}\right)$, HatchSlack $\left(\mathrm{C}_{4}\right)$ y Metabolismo Ácido de las Crasuláceas (CAM) (Ehleringer, 1979). La discriminación de ${ }^{13} \mathrm{C}$, y su fijación proporcional con respecto a ${ }^{12} \mathrm{C}$, es diferente según la vía fotosintética considerada, de manera que los valores de plantas $\mathrm{C}_{3}$ oscilan entre los $-22 \%$ y $-30 \%$ o (media $=-26,5 \%$ ); los de plantas $\mathrm{C}_{4}$, entre los $-10 \%$ y $-14 \%$ (media $=-12 \%$ ); y los de las especies vegetales CAM poseen una media de -19\%o (Ambrose y De Niro, 1986; Cerling et al., 1997). Las plantas terrestres obtienen nitrógeno del suelo, de la atmósfera (por fijación de $\mathrm{N}_{2}$ mediante relaciones simbióticas con bacterias diazótrofas) y de la descomposición de materia orgánica, con valores que oscilan entre los $-8 \%$ y los $18 \%$ y con un valor medio de 3\%o (Pate, 1994; Kelly, 2000).

La distribución de plantas $\mathrm{C}_{3}$ y $\mathrm{C}_{4}$ está determinada principalmente por la temperatura y la humedad del ambiente (Ehleringer, 1979). Las plantas $C_{4}$ poseen mayores abundancias en zonas altitudinales más bajas (Tieszen et al., 1979; Cavagnaro, 1988; Szpak et al., 2013). Diversas investigaciones desarrolladas en el área andina han demostrado la variabilidad de los valores de $\delta^{13} \mathrm{C}$ en función de la altitud, la disponibilidad de agua y la radiación solar (Szpak et al., 2013; Grant, 2014; Samec, 2014; Samec et al., 2017).
Estudios realizados en diferentes partes del mundo señalan una correlación negativa de los valores de $\delta^{15} \mathrm{~N}$ medidos en suelos, plantas y animales herbívoros con las precipitaciones medias anuales o la humedad (Pate, 1994; Amundson et al., 2003; Szpak et al., 2013; Samec, 2015). Diversos investigadores explicaron la relación entre los valores de $\delta^{15} \mathrm{~N}$ medidos en herbívoros y la disponibilidad de agua, tanto por las características fisiológicas de estos animales (Ambrose, 1986) como en función de su dieta (Murphy y Bowman, 2006; Hartman, 2011).

\section{ÁREA DE ESTUDIO: SITIOS ARQUEOLÓGICOS Y AMBIENTE}

La quebrada de Humahuaca es un valle andino localizado en el centro-este de la provincia de Jujuy (Argentina). La puna constituye su límite norte y oeste, y las selvas orientales, su límite este y sur, por lo que esta quebrada y sus tributarias conectan diversos ambientes (Hernández Llosas, 2002). El área de estudio corresponde a sus nacientes, específicamente a las quebradas de Chaupi Rodeo y Cóndor, donde se ubican los sitios arqueológicos Antumpa y Cóndor II (Figura 1).

La vegetación corresponde a las provincias fitogeográficas Prepuneña, Puneña y Altoandina, que comprenden distintos escalones altitudinales (Cabrera, 1971; Cabrera y Willink, 1973). En la quebrada de Chaupi Rodeo están representadas las dos primeras, y en la de Cóndor, la Puneña y Altoandina.

En la provincia Prepuneña domina la estepa arbustiva xerófila (e.g., Gochnatia glutinosa, Aphyllocladus spartioides y Baccharis boliviensis), cactáceas como Echinopsis atacamensis subsp.
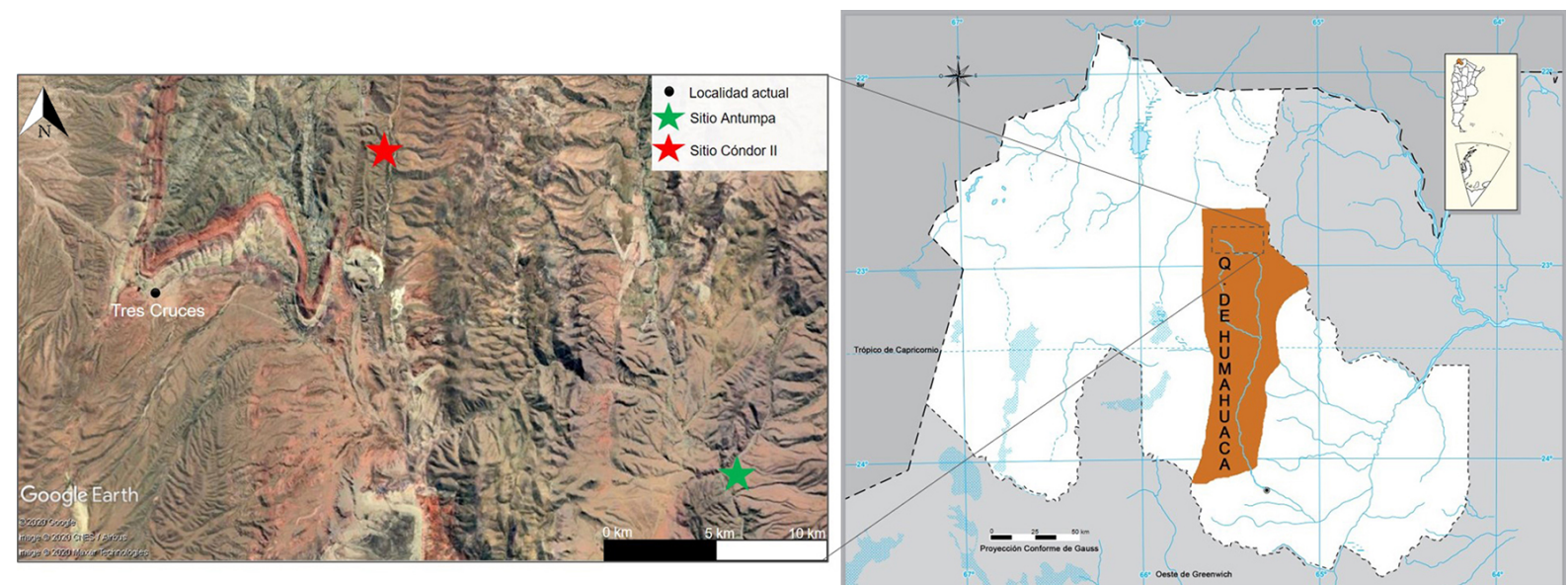

Figura 1. Localización del área de estudio y sitios arqueológicos analizados. Referencias: Q. de Humahuaca = Quebrada de Humahuaca. 
Pasacana, gramíneas (e.g., Aristida adscensionis y Jarava plumosula) y arbustos como Prosopis ferox (Cabrera, 1971; Cabrera y Willink, 1973; Ruthsatz y Movia, 1975). La vegetación de la provincia Puneña (3400-4000 msnm) se caracteriza por el predominio de la estepa arbustiva (e.g., B. boliviensis, Fabiana densa y Ephedra breana), además de estepa herbácea y vegas compuestas por tolares, hierbas y gramíneas (e.g., Nassella arcuata y Aristida asplundii) (Cabrera, 1971; Cabrera y Willink, 1973; Ruthsatz y Movia, 1975). En las quebradas de acceso a la puna existen matorrales de Adesmia inflexa, y en los fondos de valle y cuerpos de agua de la puna, tolares de Parastrephia sp. (Ruthsatz y Movia, 1975). La provincia Altoandina comprende sectores elevados (>4000 msnm) de pastizales abiertos con gramíneas bajas (e.g., Festuca orthophylla, Nassella caespitosa y Deyeuxia curvula), mezcladas con arbustos como Baccharis tola, Ephedra chilensis, P. lucida y matorrales de Polylepis tomentella (Ruthsatz y Movia, 1975).

Antumpa se localiza en la confluencia del arroyo Chaupi Rodeo con el río Grande, a 3300 msnm (Leoni et al., 2012). Es un asentamiento disperso que comprende estructuras circulares y montículos que habrían funcionado como áreas habitacionales domésticas distribuidas entre otras de forma rectangular y cuadrangular vinculadas con prácticas agrícolas y pastoriles (Leoni et al., 2012). El registro zooarqueológico presenta buenas condiciones de preservación (perfiles de meteorización bajos-moderados y ausencia de preservación diferencial de partes esqueletales) y se caracteriza por la elevada representación de artiodáctilos (34,8\%) y camélidos (30,4\%). Se registró una importante incidencia de la caza de camélidos silvestres, ya que vicuñas y guanacos en conjunto presentaron mayores porcentajes

\begin{tabular}{|l|l|c|l|l|l|}
\hline \multicolumn{1}{|c|}{ Código } & \multicolumn{1}{|c|}{ Sitio } & \multicolumn{1}{|c|}{ Fecha AP } & \multicolumn{1}{|c|}{ Calibración $\mathbf{2} \sigma^{*}$} & \multicolumn{1}{|c|}{$\delta^{13} \mathbf{C}$} & Material \\
\hline LP-3376 & Antumpa & $1980 \pm 60$ & 59 AC-219 DC & $-24 \%$ & Carbón \\
\hline UA-43082 & Antumpa & $1606 \pm 30$ & $\begin{array}{l}419-549 \text { DC }(p=.91) \\
557-576 \text { DC }(p=.08)\end{array}$ & $-22,1 \%$ Paja \\
& & & quemada \\
\hline LP-2122 & Antumpa & $1360 \pm 80$ & $586-892$ DC & $-24 \%$ & Carbón \\
\hline LP-1996 & Antumpa & $1330 \pm 70$ & $\begin{array}{l}639-893 \text { DC }(p=.99) \\
940-952 \text { DC }(p=.01)\end{array}$ & $-24 \%$ & Carbón \\
\hline LP-2872 & Cóndor II & $1130 \pm 70$ & $\begin{array}{l}773-821 \text { DC }(p=.07) \\
829-1050 \text { DC }(p=.85) \\
1083-1145 \text { DC }(p=.06)\end{array}$ & $-24 \%$ & Carbón \\
\hline LP- 2842 & Cóndor II & $960 \pm 50$ & $1026-1215$ DC & $-24 \%$ Carbón \\
\hline
\end{tabular}

* Realizados con el programa CALIB 8.1 html (Stuiver et al., 2020), usando la curva de calibración SHCal20 (Hogg et al., 2020).

Tabla 1. Fechados radiocarbónicos de los conjuntos de procedencia de los materiales analizados. de representación que la especie doméstica (Ilama) (Hernández, 2019). La arqueofauna analizada con métodos isotópicos proviene de contextos fechados entre ca. 2000-1300 años AP (Tabla 1) (Leoni et al., 2012; Leoni et al., 2015; J. B. Leoni comunicación personal, 2016).

Cóndor II se ubica en el tramo superior de la quebrada de Cóndor, sobre la terraza localizada en la margen derecha del arroyo homónimo, a 3810 msnm (Leoni et al., 2014). El sitio correspondería a un poblado disperso o semiconglomerado pequeño afectado por procesos posdepositacionales. Las ocupaciones humanas identificadas fueron datadas entre ca. 1100-900 años AP (Tabla 1) (Leoni et al., 2014). El material zooarqueológico evidenció buenos niveles de preservación (perfil de meteorización bajo y ausencia de preservación diferencial de partes esqueletales). Los restos de artiodáctilos y camélidos componen más del $90 \%$ del total de la muestra analizada. Las especies silvestres de camélidos (vicuñas y guanacos) mostraron frecuencias mayores que los animales domésticos (Hernández, 2019).

\section{MATERIALES Y MÉTODOS}

Se realizaron mediciones de los valores de $\delta^{13} \mathrm{C}$ y $\delta^{15} \mathrm{~N}$ sobre colágeno óseo en 11 especímenes arqueológicos procedentes de los sitios Antumpa ( $\mathrm{n}=$ 6) y Cóndor II $(n=5)$. Se seleccionaron elementos óseos con centros de osificación fusionados (i.e., camélidos adultos) y con escaso tejido esponjoso para evitar la erosión química y la presencia de partículas posdepositacionales que contaminaran las muestras (Ambrose, 1993). Se escogieron especímenes que no se fragmentaran con facilidad, con estadios de meteorización entre 0 y 2 (sensu Behrensmeyer, 1978) y sin termoalteraciones, presuponiendo que poseerían colágeno mejor preservado. La asignación taxonómica a nivel de especie se realizó morfológica y métricamente empleando criterios de tamaño y forma (Hernández, 2019).

La extracción del colágeno óseo fue 
realizada por los autores en el Laboratorio de Ecología Evolutiva Humana (LEEH-UNCPBACONICET) siguiendo el protocolo definido por Ambrose (1990). Los especímenes fueron limpiados con agua desionizada, secados, molidos y sometidos a un proceso de eliminación de lípidos consistente en sumergir las muestras en una solución de cloroformo-metanol por 48 horas. A continuación, se realizó la desmineralización ósea con ácido clorhídrico $\left(0.6 \mathrm{~N} \mathrm{HCl}\right.$ a $\left.4{ }^{\circ} \mathrm{C}\right)$. Posteriormente se remojó el colágeno pseudomorfo en agua doble destilada para llevar el $\mathrm{PH}$ a la neutralidad y se lo trató con $5 \% \mathrm{KOH}$ para remover contaminantes orgánicos durante dos o tres días. Luego, las muestras fueron sumergidas en solvente por otras 48 horas, secadas y pesadas. La fracción química resultante fue gelatinizada en cinco mililitros de agua acidificada por 24 horas a $120{ }^{\circ} \mathrm{C}$. Finalmente, las fases agua soluble e insoluble fueron separadas por filtración, y la fase agua-soluble fue liofilizada y pesada para obtener el rendimiento final de colágeno.

Los valores de $\delta^{13} \mathrm{C}$ y $\delta^{15} \mathrm{~N}$ fueron medidos con un espectrómetro de masas modo de flujo continuo en el Stable Isotope Ratio Facility for Environmental Research (SIRFER, University of Utah). Los datos son reportados en la notación estándar $\delta$ (delta) en unidades de partes por mil (\%o), que representa diferencias relativas en la tasa de isótopos estables de una muestra con respecto a un material estándar [e.g., $\delta^{13} \mathrm{C}(\%)=\left(\mathrm{R}_{\text {muestra }} / \mathrm{R}_{\text {estándar }}-1\right)$, donde $\mathrm{R}$ representa la tasa del isótopo pesado con respecto al liviano $\left.\left({ }^{13} \mathrm{C} /{ }^{12} \mathrm{C}\right)\right]$. Los valores de $\delta^{13} \mathrm{C}$ se expresan con respecto a la escala Vienna Peedee Belemnite (V-PDB) y los de $\delta^{15} \mathrm{~N}$, con respecto a la escala AIR, utilizando USGS40 y USGS41. Las muestras fueron analizadas junto con dos materiales de referencia primaria internos de SIRFER (PLRMs: UU-CN-1 $\delta^{13} \mathrm{C}_{\mathrm{VPDB}}=+23,96 \%, \delta^{15} \mathrm{~N}_{\mathrm{AIR}}=+49,63 \%$; UU-CN-2 $\delta^{13} C_{\text {VPDB }}=-28,18 \%, \delta^{15} \mathrm{~N}_{\text {AIR }}=-4,56 \%$ ) y a un material de referencia secundario (SLRM $\delta^{13} \mathrm{C}_{\mathrm{VPDB}}=-27,41 \%$, $\delta^{15} \mathrm{~N}_{\mathrm{AIR}}=-0,4 \%$ ) como control de calidad. La incertidumbre analítica para ambos isótopos fue menor al 0,2\%. Los porcentajes de elementos $\mathrm{C}$ y $\mathrm{N}$ y la proporción $\mathrm{C}: \mathrm{N}$ fueron usados para evaluar la calidad del colágeno extraído y descartar alteraciones diagenéticas (De Niro, 1985; Ambrose, 1990).

Los valores isotópicos fueron analizados con el software PAST 4.02 (Hammer et al., 2001) mediante el uso de estadística descriptiva y univariada. Se realizaron reconstrucciones de la dieta potencial de los camélidos utilizando las distribuciones de probabilidad reales de las contribuciones relativas de especies vegetales $C_{3}$ y el porcentaje $C_{4} y$ CAM de manera conjunta (i.e., de dos fuentes) (ver Samec, 2015), a los valores de isótopos del colágeno. Para estimar dichas contribuciones se ajustaron modelos de mezcla lineal en un marco bayesiano utilizando el paquete SIAR para R (Parnell et al., 2010). Si bien los modelos bayesianos son normalmente utilizados en sistemas indeterminados (i.e., cuando hay un número de marcadores isotópicos menor al número de fuentes de alimentación + 1), y en nuestro caso tenemos un sistema determinado (dos fuentes $-\mathrm{C}_{3}$ y $\mathrm{C}_{4}-$ y dos marcadores $-\delta^{13} \mathrm{C}$ y $\delta^{15} \mathrm{~N}-$ ); ante la falta de datos sobre ecología isotópica, estos presentan la ventaja de permitir la incorporación de varias fuentes de incertidumbre como son múltiples datos para el consumidor (e.g., muestras de camélidos individuales), múltiples fuentes potenciales de alimentos y su variabilidad isotópica (promedio $\pm \mathrm{DE}$ ), y los factores de corrección de nivel trófico y su variabilidad asociada (promedio \pm DE). Para su implementación se utilizaron los isótopos de $\delta^{13} \mathrm{C}$ y $\delta^{15} \mathrm{~N}$ medidos en los restos óseos de camélidos arqueológicos de los sitios estudiados. Cada modelo se ejecutó durante 50.000 iteraciones, descartando las primeras 5000 y sin dependencia de la concentración (asumiendo que todas las fuentes de $\mathrm{C} / \mathrm{N}$ son iguales). No se agregó información externa previa (prior) para guiar los modelos. Los factores de corrección tróficos para estimar la dieta de los ungulados fueron de $3,4 \pm 1,0 \%$ para $\delta^{15} \mathrm{~N}$ y $5,0 \pm 1,0 \%$ para los valores de $\delta^{13} \mathrm{C}$ (Koch et al., 1994; Post, 2002). Se calcularon el valor medio de la dieta de los camélidos de ambos sitios y sus desvíos estándar (DE). Se utilizaron los valores medios de $\delta^{13} \mathrm{C}$ de la vegetación $\mathrm{C}_{3}$ y $\mathrm{C}_{4}+\mathrm{CAM}$ de la puna jujeña generados por Samec (2015) para altitudes inferiores a los 3900 msnm, se estimaron los respectivos desvíos estándar (DE) y los valores medios y DE para $\delta^{15} \mathrm{~N}$ en base a los datos generados por dicha investigación.

\section{RESULTADOS}

Los valores de $\delta^{13} \mathrm{C}$ y $\delta^{15} \mathrm{~N}$ de camélidos arqueológicos, sus porcentajes elementales y la relación $\mathrm{C}: \mathrm{N}$ son presentados en la Tabla 2. En todos los casos, la proporción $\mathrm{C}: \mathrm{N}$ se encuentra dentro del rango de una señal isotópica primaria, por lo cual se descarta la incidencia de procesos diagenéticos que afecten la composición del colágeno preservado (De Niro, 1985). Los valores de $\delta^{13} \mathrm{C}$ de los 
especímenes varían entre $-18,3 \%$ y $-14,0 \%$, con una media de $-16,2 \%$; y los de $\delta^{15} \mathrm{~N}$, entre $6,4 \%$ y $8,9 \%$, con una media de $7,7 \%$.

La distribución de los datos indica que las especies de camélidos representadas poseen composiciones isotópicas similares, en tanto se registran escasas diferencias, aunque por el momento el tamaño de la muestra no permite realizar comparaciones estadísticas (Figura 2). En cuanto a los $\delta^{13} \mathrm{C}$, los cuatro especímenes identificados como llamas presentan un valor medio de - $17 \%$, el único espécimen de guanaco exhibe una composición isotópica de $-17,1 \%$, el espécimen asignado a vicuña posee un valor de $-17,5 \%$, y los cinco especímenes atribuibles a camélidos de gran porte (Lama sp.) presentan una composición media de $-16 \%$. Los datos de $\delta^{15} \mathrm{~N}$ señalan valores medios de $7,4 \%$ para los cuatro especímenes identificados como llamas, una composición isotópica de 6,4\%o para el de guanaco y de $7,5 \%$ para el espécimen de vicuña, y valores medios de $7,9 \%$ para los cinco especímenes atribuibles a camélidos grandes. Las mayores diferencias en $\delta^{15} \mathrm{~N}$ se registraron entre

\begin{tabular}{|l|l|l|l|l|l|l|l|}
\hline \multicolumn{1}{|c|}{ Código* } & Elemento & Taxón & $\delta^{\mathbf{1 3}} \mathbf{C} \%$ & $\delta^{\mathbf{1 5}} \mathbf{N} \mathbf{\%}$ & $\mathbf{N} \%$ & $\mathbf{C} \%$ & $\mathbf{C}: \mathbf{N}$ \\
\hline ANT145 & Húmero & Llama & $-15,7$ & 7,5 & 14,9 & 41,0 & 3,0 \\
\hline CII170 & Húmero & Lama sp. & $-17,1$ & 7,1 & 16,1 & 43,5 & 2,9 \\
\hline CII282 & $1^{\circ}$ Falange & Llama & $-18,2$ & 7,9 & 14,1 & 38,6 & 2,9 \\
\hline CII352 & Metatarso & Vicuña & $-17,5$ & 7,5 & 13,7 & 38,2 & 3,0 \\
\hline CII357 & Radio-ulna & Lama sp. & $-18,3$ & 8,1 & 15,4 & 41,7 & 2,9 \\
\hline CII389 & $1^{\circ}$ Falange & Guanaco & $-17,1$ & 6,4 & 14,9 & 41,4 & 3,0 \\
\hline ANT392 & Metatarso & Lama sp. & $-14,0$ & 8,8 & 14,6 & 40,0 & 2,9 \\
\hline ANT490 & $1^{\circ}$ Falange & Llama & $-17,3$ & 7,6 & 14,3 & 39,9 & 3,0 \\
\hline ANT989 & $1^{\circ}$ Falange & Llama & $-16,8$ & 6,7 & 14,6 & 40,3 & 3,0 \\
\hline ANT2996 & H. largo & Lama sp. & $-16,7$ & 7,0 & 12,8 & 35,2 & 2,9 \\
\hline ANT3042 & Metapodio & Lama sp. & $-14,3$ & 8,9 & 15,3 & 41,9 & 2,9 \\
\hline
\end{tabular}

*Referencias: ANT = Antumpa; CII = Cóndor II.

Tabla 2. Valores de $\delta^{13} \mathrm{C}$ y $\delta^{15} \mathrm{~N}$ y proporción C: $\mathrm{N}$ de la muestra analizada.

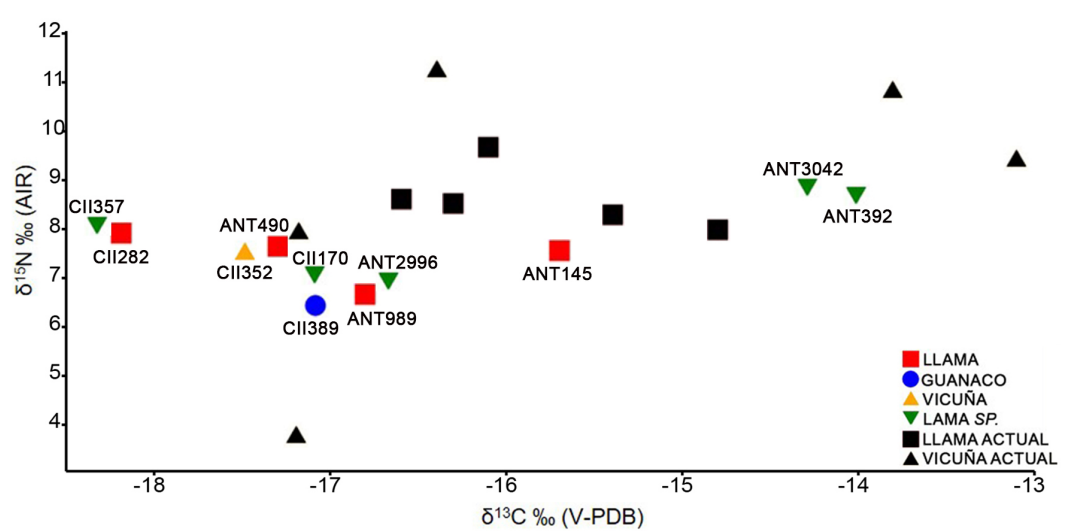

Figura 2. Valores de $\delta^{13} \mathrm{C}$ y $\delta^{15} \mathrm{~N}$ de la muestra arqueológica estudiada y de camélidos modernos (<3900 msnm) estudiados por Samec $(2014,2015)$. guanaco $(6,4 \%)$ y llamas $(7,9 \%)$, y entre vicuña $(7,5 \%)$ y guanaco $(6,4 \%)$. De la muestra analizada destacan dos especímenes pertenecientes a camélidos grandes (ANT392 y ANT3042), cuyos valores de $\delta^{13} \mathrm{C}$ y de $\delta^{15} \mathrm{~N}$ son mayores al resto de la muestra (Figura 2). Estos se distribuyen próximos a los valores de dos vicuñas y una llama moderna (ver Samec, 2015) procedentes de altitudes similares $(<3900$ msnm).

Considerando que la bibliografía para el área sugiere la existencia de variación de los valores de $\delta^{13} \mathrm{C}$ y $\delta^{15} \mathrm{~N}$ en función de la altitud (Fernández y Panarello, 1999-2001; Yacobaccio et al., 2009, 2010; Samec, 2014, 2015; Samec et al., 2014), se decidió comparar cualitativamente las muestras de Antumpa (3300 msnm) y Cóndor II (3810 msnm), para establecer si existen diferencias en la dieta de los camélidos dentro del área de estudio. Los resultados obtenidos indican distintos valores de $\delta^{13} \mathrm{C}$ entre los camélidos de ambos sitios (Figura 3a). Los especímenes de Antumpa registran valores mayores (entre $-14 \%$ y $-17,3 \%$ ) que los de Cóndor II (entre $-17,1 \%$ y $-18,3 \%$ ), sin que se observe superposición entre ellos. Además, la muestra de Antumpa exhibe una mayor variabilidad en la composición isotópica del carbono.

Para determinar si estas diferencias obedecen a un mayor aporte de especies $\mathrm{C}_{4}$ a la dieta de los camélidos de Antumpa se empleó un modelo de mezcla lineal en un marco bayesiano para estimar los porcentajes de ingesta de los vegetales de los camélidos representados en ambos sitios. Los resultados indican que la dieta de los camélidos de Antumpa estaría conformada por proporciones mayores de vegetales $\mathrm{C}_{4} \mathrm{y}$ CAM (entre $22 \%$ y $75 \%$, media $=44 \%$ ) que la de Cóndor II (entre $2 \%$ y $62 \%$, media $=30 \%$ ) (Tabla 3).

En cuanto a los valores de $\delta^{15} \mathrm{~N}$, no se identificó variación en relación con la altitud, en tanto la comparación de las muestras de Antumpa y Cóndor 
II revela composiciones isotópicas similares entre los camélidos de ambos conjuntos (Figura 3b).

Finalmente, indagamos si los valores de $\delta^{13} \mathrm{C}$ y $\delta^{15} \mathrm{~N}$ de la dieta de los camélidos arqueológicos podrían explicarse por el consumo de especies vegetales presentes en las quebradas del norte de Humahuaca. Actualmente no contamos con información isotópica de la vegetación de las quebradas de Chaupi Rodeo y Cóndor, por lo que recurrimos a los datos generados por otros investigadores para otra quebrada tributaria a la de Humahuaca, Inca Cueva (Samec, 2015), localizada aproximadamente a unos $14 \mathrm{~km}$ de nuestra área de estudio.

Los resultados muestran que los valores medios de las dietas de los camélidos de Antumpa $\left(\delta^{13} \mathrm{C}=\right.$ $-20,8 \%$, $\delta^{15} \mathrm{~N}=4,3 \%$ ) y Cóndor II $\left(\delta^{13} \mathrm{C}=-22,6 \%\right.$,
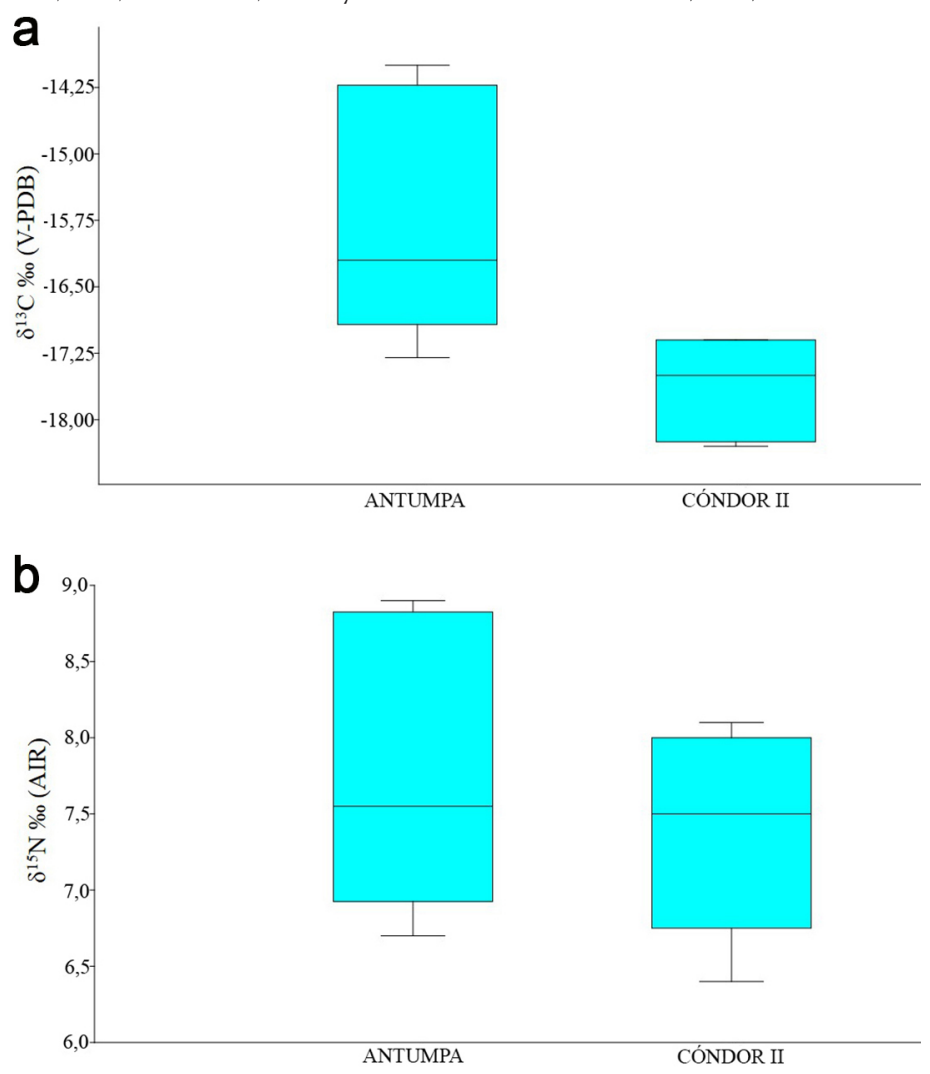

Figura 3. Composición isotópica de camélidos por sitio arqueológico y nivel altitudinal. a- Valores de $\delta^{13} \mathrm{C}$. b- Valores de $\delta^{15} \mathrm{~N}$.

\begin{tabular}{|c|c|c|c|c|}
\hline \multirow[b]{2}{*}{ Sitio } & \multirow[b]{2}{*}{$\begin{array}{c}\text { Tipo } \\
\text { Fotosintético }\end{array}$} & \multicolumn{3}{|c|}{ Modelaje Bayesiano SIAR } \\
\hline & & Media & Intérvalo & $\begin{array}{c}\text { Desvío } \\
\text { estándar }\end{array}$ \\
\hline \multirow{2}{*}{ Antumpa } & $\mathrm{C}_{3}$ & 0,56 & $0,25-0,78$ & 0,06 \\
\hline & $\mathrm{C}_{4}+\mathrm{CAM}$ & 0,44 & $0,22-0,75$ & 0,06 \\
\hline \multirow{2}{*}{ Cóndor II } & $\mathrm{C}_{3}$ & 0,70 & $0,38-0,98$ & 0,07 \\
\hline & $\mathrm{C}_{4}+\mathrm{CAM}$ & 0,30 & $0,02-0,62$ & 0,07 \\
\hline
\end{tabular}

Tabla 3. Resultados del modelaje SIAR. Contribuciones proporcionales de los tipos fotosintéticos $\mathrm{C}_{3}$ y $\mathrm{C}_{4}+\mathrm{CAM}$ a la dieta de los camélidos de Antumpa y Cóndor II. $\delta^{15} \mathrm{~N}=4 \%$ o) se encuentran dentro del rango de variación estimado para las distintas comunidades vegetales modernas representadas en esta última quebrada (ver Samec, 2015).

\section{DISCUSIÓN Y CONCLUSIONES}

Los resultados de $\delta^{13} \mathrm{C}$ y $\delta^{15} \mathrm{~N}$ obtenidos para camélidos arqueológicos de las nacientes de la quebrada de Humahuaca son afines a los reportados para sitios del Holoceno tardío de la Puna Seca jujeña y a los valores registrados para camélidos modernos procedentes de niveles altitudinales similares ( 3900 msnm) (Fernández y Panarello, 19992001; Samec, 2015).

Este estudio muestra diferencias entre los camélidos de Antumpa y Cóndor II en cuanto a $\delta^{13} \mathrm{C}$, y similitudes en los valores de $\delta^{15} \mathrm{~N}$. Resulta probable que la distribución de plantas $\mathrm{C}_{3}$ y $\mathrm{C}_{4}$ en función de la altitud, señalada por distintas investigaciones, explique parcialmente la tendencia registrada en los valores de $\delta^{13} \mathrm{C}$ (Tieszen et al., 1979; Fernández y Panarello, 1999-2001; Szpak et al., 2013; Samec et al., 2017). Las plantas $C_{4}$ presentarían mayores abundancias en zonas de menor altitud con bajos índices de humedad y mayor temperatura (Tieszen et al., 1979). Este hecho extrapolado al área de estudio explicaría por qué los valores de $\delta^{13} \mathrm{C}$ de los camélidos de Cóndor II son más negativos y los de Antumpa más altos. La localización de Cóndor II a 3810 msnm implicaría el pastoreo de camélidos y la caza de estos ungulados cerca del límite de los 3900 msnm, por sobre el cual las especies vegetales $C_{4}$ no abundan (Samec et al., 2017). En cambio, el mayor aporte de especies $C_{4}$ en la dieta de los camélidos de Antumpa obedecería no solo a la distribución y abundancia natural de los distintos tipos fotosintéticos de plantas en el área, sino que otros factores, como el pastoreo en zonas de menor altitud, podrían incidir en la señal isotópica registrada. La presencia de dos especímenes de gran tamaño procedentes del sitio Antumpa con valores de $\delta^{13} \mathrm{C}$ y $\delta^{15} \mathrm{~N}$ elevados lleva 
a plantearnos la posibilidad de que su composición isotópica se deba, en caso de tratarse de llamas, a prácticas de manejo de rebaños como el uso de zonas de pasturas naturales con mayor preponderancia de vegetales $\mathrm{C}_{4}$. Cabe destacar que Fernández y Panarello (1999-2001) registraron un valor de $\delta^{13} \mathrm{C}$ en una Ilama actual $(<4000 \mathrm{msnm})$, similar al de los dos especímenes de Antumpa, que fue interpretado como resultado de la probable intervención humana mediante su alimentación con plantas $\mathrm{C}_{4}$ como el maíz (Yacobaccio et al., 2009). Si bien este escenario también podría explicar los valores de los dos especímenes de Antumpa, no contamos con evidencia contextual para confirmarlo.

Los valores de $\delta^{15} \mathrm{~N}$ no mostraron correlación con la altitud, a diferencia de otras investigaciones (Samec, 2014, 2015; Samec et al., 2018). Esto podría ser el resultado del tamaño de la muestra analizada, de la escasa diferencia altitudinal existente entre los sitios en comparación con aquella analizada en otras áreas (Szpak et al., 2013), o reflejar la ausencia real de variabilidad en la composición isotópica del nitrógeno entre ambos escalones altitudinales en el área de estudio. No obstante, se ha señalado que los factores ambientales y climáticos locales inciden fuertemente en los valores de $\delta^{15} \mathrm{~N}$ de especies vegetales, por lo que su actuación podría ser responsable de la falta de asociación entre estos y la altitud (Heaton, 1987; Ambrose, 1991; Samec, 2015). En consecuencia, dicha correlación tampoco se observaría en el siguiente nivel de la cadena trófica (i.e., herbívoros). Asimismo, como fuera mencionado anteriormente, las muestras estudiadas corresponden a diferentes lapsos temporales dentro del Holoceno tardío (Antumpa 1900-1300 AP; Cóndor II 1100-960 AP), por lo que los resultados obtenidos también podrían reflejar variaciones temporales en la composición isotópica del carbono, y su ausencia en el caso del nitrógeno producto de la existencia de condiciones locales (humedad y temperatura) similares para las quebradas de Chaupi Rodeo y de Cóndor a lo largo del segmento temporal analizado. Actualmente, no contamos con datos suficientes para precisar el alcance de esta posible fuente de variabilidad, ya que se requieren investigaciones regionales que utilicen distintos proxy paleoclimáticos.

Otro aspecto a destacar es la escasa variabilidad registrada en la composición isotópica de los restos pertenecientes a las distintas especies de camélidos identificadas por criterios de tamaño y forma. Si bien la cantidad de especímenes estudiados podría tener una incidencia directa, estudios realizados con muestras más numerosas procedentes de la puna jujeña señalan que la composición isotópica de las distintas especies silvestres y domésticas de camélidos no refleja diferencias en el consumo de plantas $\mathrm{C}_{3}$ y $\mathrm{C}_{4}$ (Samec et al., 2014; Samec, 2015). Por el contrario, sí fueron identificadas en camélidos procedentes de otros sectores del NOA (Izeta et al., 2009; Dantas, 2012). En relación con $\delta^{15} \mathrm{~N}$, tampoco se registraron variaciones isotópicas entre llamas y vicuñas, que sin embargo fueron identificadas en investigaciones basadas en el análisis de mayor cantidad de especímenes, y que podrían explicarse por diferencias en la dieta de animales silvestres y domésticos o por sus características fisiológicas (Samec, 2015; Samec et al., 2018). En cambio, el único valor de $\delta^{15} \mathrm{~N}$ correspondiente a vicuña presentó diferencias respecto del único valor disponible para guanaco. Nuevos estudios que amplíen la muestra estudiada son fundamentales para corroborar la presencia de composiciones isotópicas de $\delta^{15} \mathrm{~N}$ diferentes entre las especies silvestres, y establecer sus posibles causas (hábitos territoriales y/o alimenticios).

Si bien es necesario corroborar esta tendencia preliminar incrementando los datos disponibles, la falta de variación isotópica aquí registrada entre camélidos silvestres y domésticos podría indicar un patrón de movilidad como el propuesto por Samec y colaboradores (2018), que habría permitido a los grupos de la región desarrollar conjuntamente actividades de caza y pastoreo en las mismas áreas.

Los valores de $\delta^{13} \mathrm{C}$ y $\delta^{15} \mathrm{~N}$ de camélidos arqueológicos de las nacientes de la quebrada de Humahuaca son consistentes con la señal isotópica de la vegetación típica de esta zona. Por ende, las áreas de caza no se habrían encontrado demasiado alejadas de los sitios y el territorio de pastoreo no incluiría cotas altitudinales muy superiores a los $3900 \mathrm{msnm}$. La información zooarqueológica disponible refuerza esta hipótesis. La amplia representación de partes esqueletales y la frecuencia de huellas, fracturas y termoalteraciones muestra una explotación integral de camélidos orientada mayormente al aprovechamiento cárnico de animales juveniles (Hernández, 2019). Para los camélidos silvestres esto habría implicado el traslado del animal prácticamente completo desde un área de caza no demasiado alejada hasta el sitio, con su consiguiente procesamiento y consumo en el lugar. 
El análisis isotópico presentado, pese a constituir un primer acercamiento al estudio de la variabilidad de $\delta^{13} \mathrm{C}$ y $\delta^{15} \mathrm{~N}$ de camélidos silvestres y domésticos de sitios del norte de la quebrada de Humahuaca, contribuye a evaluar aspectos de su explotación y manejo. Los resultados deberán corroborarse en futuras investigaciones mediante el incremento de las muestras analizadas, lo que posibilitará profundizar el conocimiento del uso de estos ungulados en el área durante el Holoceno tardío.

\section{Agradecimientos}

Agradecemos a M. I. Hernández Llosas y a J. B. Leoni por facilitarnos las muestras arqueológicas analizadas, y a G. L. L`Heureux y a los evaluadores anónimos por los comentarios, que contribuyeron a mejorar este manuscrito. Este trabajo se realizó en el marco de la beca doctoral otorgada por CONICET a la primera autora.

\section{REFERENCIAS CITADAS}

Ambrose, S. H. (1986). Stable carbon and nitrogen isotope analysis of human and animal diet in Africa. Journal of Human Evolution, 15, 707-731. https://doi.org/10.1016/ S0047-2484(86)80006-9

Ambrose, S. H. (1990). Preparation and characterization of bone and tooth collagen for isotopic analysis. Journal of Archaeological Science, 17, 431-451. https://doi. org/10.1016/0305-4403(90)90007-R

Ambrose, S. H. (1991). Effects of diet, climate and physiology on nitrogen isotope abundances interrestrial foodwebs. Journal of Archaeological Science, 18, 293317. https://doi.org/10.1016/0305-4403(91)90067-Y

Ambrose, S. H. (1993). Isotopic analysis of paleodiets: metodological and interpretive considerations. En M. K. Sandford (Ed.), Investigations of ancient human tissue. Chemical Analyses in Anthropology (pp. 59-130). Gordon and Breach Science Publishers.

Ambrose, S. H. y De Niro, M. J. (1986). Reconstruction of African human diet using bone collagen carbon and nitrogen isotope ratios. Nature, 319, 321-324. https:// doi.org/10.1038/319321a0

Amundson, R., Austin, A. T., Schuur, E. A. G., Yoo, K., Matzek, V., Kendall, C., Uebersax, A., Brenner, D. y Baisden, W. T. (2003). Global patterns of the isotopic composition of soil and plant nitrogen. Global Biogeochemical cycles, 17(1), 1031. https://doi. org/10.1029/2002GB001903
Balasse, M., Ambrose, S. H., Smith, T. D. y Price, D. (2002). The seasonal mobility model for prehistoric herders in the south-western Cape of South Africa assessed by isotopic analysis of sheep tooth enamel. Journal of Archaeological Science, 29, 917-932. https:// doi.org/10.1006/jasc.2001.0787

Berhensmeyer, A. K. (1978). Taphonomic and ecologic information from bone weathering. Paleobiology, 4, 150-62. https://doi.org/10.1017/S0094837300005820

Bonavia, D. (2008). The South American Camelids. An expanded and corrected edition. The Cotsen Institute of Archaeology Press.

Borgnia, M., Vilá, B. L. y Cassini, M. H. (2010). Foraging ecology of Vicuña, Vicugna, in dry Puna of Argentina. Small Ruminant Research, 88, 44-53. https://doi. org/10.1016/j.smallrumres.2009.11.009

Cabrera, A. L. (1971). Fitogeografía de la república Argentina. Boletín de la Sociedad Argentina de Botánica, 1-2(XIV), 1-50.

Cabrera, A. L. y Willink, A. (1973). Biogeografía de América Latina. Serie de Biología, monografía 13. Secretaría General de la Organización de los Estados Americanos.

Cavagnaro, J. B. (1988). Distribution of $\mathrm{C}_{3}$ and $\mathrm{C}_{4}$ grasses at different altitudes in a temperate arid region of Argentina. Oecología, 76, 273-277.

Cerling, T. E., Harris, J. M., MacFadden, B. J., Leakey, M. G., Quadek, J., Eisenmann, V. y Ehleringer, J. R. (1997). Global vegetation change through the Miocene/ Pliocene boundary. Nature, 389, 153-158. https://doi. org/10.1038/38229

Dantas, M. (2012). Identificación interespecífica de camélidos en el valle de Ambato (Catamarca, Argentina). Una aproximación a la problemática desde distintas líneas de análisis. Revista del Museo de Antropología, 5(1), 259-268. https://doi. org/10.31048/1852.4826.v5.n1.9197

De Niro, M. (1985). Postmortem preservation and alteration of in vivo bone collagen isotope ratios in relation to palaeodietary reconstruction. Nature, 317, 806-809. https://doi.org/10.1038/317806a0

Dufour, E., Goepfert, N., Gutiérrez León, B., Chauchat, C., Jordán, R. F. y Vásquez Sánchez, S. (2014). Pastoralism in Northern Peru during Pre-Hispanic times: insights from the Mochica period (100-800 AD) based on stable isotopic analysis of domestic camelids. PLOS ONE, 9(1), e87559. https://doi.org/10.1371/journal.pone.0087559

Ehleringer, J. R. (1979). Photosyntesis and photorespiration: biochemistry, physiology, and ecological implications. HortScience, 14(3), 217-222. 
Fernández, J. y Panarello, H. O. (1999-2001). Isótopos del carbono en la dieta de herbívoros y carnívoros de los Andes jujeños. Xama, 12-14, 71-85.

Finucane, B. C., Maita Agurto, P. e Isbell, W. H. (2006). Human and animal diet at Conchopata, Perú: stable isotope evidence for maize agriculture and animal management practices during the Middle Horizon. Journal of Archaeological Science, 33, 1766-1776. https://doi.org/10.1016/j.jas.2006.03.012

Fuchs, M. L., Cocilovo, J. A. y Varela, H. H. (2015). Análisis de la paleodieta a partir de isótopos estables del carbono y del nitrógeno en la población prehispánica de la puna de Jujuy (Argentina). Estudios Atacameños, 51, 123-135. https://doi.org/10.4067/ $\underline{\text { S0718-10432015000200008 }}$

Gallardo, F. y Yacobaccio, H. D. (2007). ¿Silvestres o domesticados? Camélidos en el arte rupestre del formativo temprano en el desierto de Atacama (norte de Chile). Boletín del Museo Chileno de Arte Precolombino, 12(2), 9-31.

Gerling, C., Doppler, T., Heyd, V., Knipper, C., Kuhn, T., Lehmann, M. F., Pike, A. W. G. y Schibler, J. (2017). High-resolution isotopic evidence of specialised cattle herding in the European Neolithic. PLOS ONE, 12(7), e0180164. https://doi.org/10.1371/journal. pone. 0180164

Gorlova, O. A., Krylovich, A., Tiunov, A. V., Khasanov, B. F., Vasyukov, D. D. y Savinetsky, A. B. (2015). Stable-isotope analysis as a method of taxonomical identification of archaeozoological material. Archaeology, Ethnology and Anthropology of Eurasia, 43(1), 110-121. https://doi.org/10.1016/j. aeae.2015.07.013

Grant, J. (2014). Manejo Económico de Camélidos en Antofagasta de la Sierra (Puna Meridional Argentina): una Aproximación Zooarqueológica e Isotópica [Tesis doctoral, Universidad de Buenos Aires].

Grant, J., Mondini, M. y Panarello, H. O. (2018). Carbon and nitrogen isotopic ecology of Holocene camelids in the Southern Puna (Antofagasta de la Sierra, Catamarca, Argentina): archaeological and environmental implications. Journal of Archaeological Science Reports, 18, 637-647. https://doi.org/10.1016/j. jasrep.2017.05.045

Grant, J. y Olivera, D. (2016). Isótopos estables, movilidad y camélidos en sociedades agropastoriles tempranas de la Puna Meridional Argentina. Arqueología Dossier, 22, 13-35. https://doi.org/10.34096\%2Farqueologia.t22. $\underline{\mathrm{n} 0.3275}$

Hammer, O., Harper, D. A. T. y Ryan, P. D. (2001). PAST: Paleontological Statistics software package for education and data analysis. Palaeontologia Electronica, 4(1), 9 pp.

Hartman, G. (2011). Are elevated $\delta^{15} \mathrm{~N}$ values in herbivores in hot and arid environments caused by diet or animal physiology? Functional Ecology, 25, 122-131. https:// doi.org/10.1111/j.1365-2435.2010.01782.x

Heaton, T. H. E. (1987). The ${ }^{15} \mathrm{~N} /{ }^{14} \mathrm{~N}$ ratios of plants in South Africa and Namibia: relationship to climate and coastal/saline environments. Oecologia, 74(2), 236-246. https://doi.org/10.1007/BF00379365

Hernández, A. (2019). Caza y Pastoreo en el sector norte y Nacientes de la Quebrada de Humahuaca Durante el Holoceno Tardío [Tesis doctoral, Universidad Nacional de Rosario].

Hernández Llosas, M. I. (2001). Arte rupestre del Noroeste argentino: orígenes y contexto de producción. En E. Berberián y A. E. Nielsen (Eds.), Historia Argentina Prehispánica, Tomo I (pp. 389-446). Brujas.

Hernández Llosas, M. I. (2002). Patrimonio cultural y desarrollo sostenible en la Quebrada de Humahuaca. Potencial y perspectivas. Cuadernos FHyCS-UNJU, 18, 125-152.

Hogg, A. G., Heaton, T. J., Hua, Q., Palmer, J. G., Turney, C. S. M., Southon, J., Bayliss, A., Blackwell, P. G., Boswijk, G., Bronk Ramsey, C., Pearson, C., Petchey, F., Reimer, P., Reimer y R., Wacker, L. (2020). SHCal20 Southern Hemisphere calibration, 0-55,000 years cal BP. Radiocarbon, 62(4), 759-778. https://doi. org/10.1017/RDC.2020.59

Izeta, A. D., Laguens, A. G., Marconetto, M. B. y Scattolin, M. C. (2009). Camelid handling in the meridional Andes during the first millennium AD: a preliminary approach using stable isotopes. International Journal of Osteoarchaeology, 19, 204-214. https://doi. org/10.1002/oa.1066

Kelly, J. F. (2000). Stable isotopes of carbon and nitrogen in the study of avian and mammalian trophic ecology. Canadian Journal of Zoology, 78, 1-27. https://doi. org/10.1139/z99-165

Killian Galván, V. A. (2018). Models for paleodietary research: Three case-studies from arid and semi-arid environments in Northwest Argentina. Journal of Archaeological Science Reports, 18, 608-616. http:// dx.doi.org/10.1016/j.jasrep.2017.09.012

Killian Galván, V. A., Seldes, V. y Nielsen, A. E. (2016). Inferencia paleodietaria en el sitio arqueológico Los Amarillos (Quebrada de Humahuaca, Jujuy, Argentina). Relaciones de la Sociedad Argentina de Antropología, 61(1), 79-99. 
Koch, P. L., Fogel, M. L. y Tuross, N. (1994). Tracing the diets of fossil animals using stable isotopes. En K. Lajtha y B. Michener (Eds.), Stable Isotopes in Ecology and Environmental Science (pp. 63-92). Blackwell Scientific Publications.

Leoni, J. B., Fabron, G., Tamburini, D., Hernández, A. y Brancatelli, C. (2014). Cóndor 2, un sitio del período de Desarrollo Regionales 1 en el sector norte de la Quebrada de Humahuaca, Jujuy. Estudios Sociales del NOA, 13, 125-146.

Leoni, J. B., Sartori, J., Fabron, G., Hernández, A. y Scarafia, G. (2012). Antumpa, un paisaje productivo del primer milenio AD en el sector norte de la Quebrada de Humahuaca. Intersecciones en Antropología, 13, 117-131.

Leoni, J. B., Tamburini, D., Fabron, G., Hernández, A. y Brancatelli, C. (2015). Análisis de un conjunto material del primer milenio D.C. del sitio Antumpa (Dpto. Humahuaca, Jujuy). El caso del sector Terraza. Anuario de Arqueología, 7, 111-130.

López Mendoza, P., Cartajena, I., Loyola, R., Núñez, L. y Carrasco, C. (2017). The Use of Hunting and Herding Spaces: Stable Isotope Analysis of Late Archaic and Early Formative Camelids in the Tulan Transect (Puna de Atacama, Chile). International Journal of Osteoarchaeology, 27, 1059-1069. https://doi. org/10.1002/oa.2631

Mengoni Goñalons, G. L. (2014). Isótopos estables en camélidos del período Tardío del Noroeste de Argentina (NOA): estrategias de uso y manejo de rebaños. Revista Chilena de Antropología, 30, 68-75. https://doi.org/10.5354/0719-1472.2015.36272

Mengoni Goñalons, G. L. y Yacobaccio, H. D. (2006). The domestication of South America Camelids: a view from south-Central Andes. En M. A. Zeder, D. Bradley, E. Emshwiller y B. D. Smith (Eds.), Documenting Domestication: Bringing Together Plants, Animals, Archaeology, and Genetics (pp. 228-243). University of California Press.

Mondini, M., Grant, J., Panarello, H. O., Samec, C., López M., P., Núñez, L. y Cartajena, I. (2019). Composición isotópica del carbono y el nitrógeno en camélidos arqueológicos de la Puna de Atacama: una comparación entre ambas vertientes de los Andes. Cuadernos del Instituto Nacional de Antropología y Pensamiento Latinoamericano, Series Especiales, 7(2), 172-181.

Murphy, B. P. y Bowman, D. M. J. S. (2006). Kangaroo metabolism does not cause the relationship between bone collagen $\delta^{15} \mathrm{~N}$ and water availability. Functional Ecology, 20, 1062-1069. https://doi. org/10.1111/j.1365-2435.2006.01186.x
Olivera, D. E. (2001). Perfil etario y rendimiento económico de Lama glama. En G. L. Mengoni Goñalons, D. E. Olivera y H. D. Yacobaccio (Eds.), El uso de los camélidos a través del tiempo (pp. 179-202). Ediciones Del Tridente.

Olivera, D. y J. Palma. (1997). Cronología y registro arqueológico en el Formativo Temprano en la región de Humahuaca. Avances en Arqueología, 3, 77-99.

Panarello, H. O. y Mondini, M. (2016). Evidencia isotópica de camélidos y vegetales modernos en la puna sur. Relevancia para la arqueología de la región. Cuadernos del Instituto Nacional de Antropología y Pensamiento Latinoamericano, 24(2), 45-62.

Parnell, A. C., Inger, R., Bearhop, S. y Jackson, A. L. (2010). Source partitioning using stable isotopes: coping with too much variation. PLOS ONE, 5, e9672. https://doi.org/10.1371/journal.pone.0009672

Pate, F. D. (1994). Bone chemistry and paleodiet. Journal of Archaeological Method and Theory, 1, 161-209. https://doi.org/10.1007/BF02231415

Post, D. (2002). Using stable isotopes to estimate trophic position: models, methods, and assumptions. Ecology, 83(3), 703-718. https://doi.org/10.2307/3071875

Puig, S., Varela, F. y Cona, M. I. (1997). Diet and abundance of the guanaco (Lama guanicoe Muller 1776) in four habitats of northern Patagonia, Argentina. Journal of Arid Environments, 36, 343-357.

Ruthsatz, B. y Movia, C. P. (1975). Relevamiento de las Estepas Andinas del Noreste de la Provincia de Jujuy. República Argentina. Fundación para la Educación, la Ciencia y la Cultura.

Samec, C. T. (2011). Perspectiva Isotópica sobre la Alimentación de Camélidos Domésticos y Silvestres de la puna Jujeña. Construyendo un marco de Referencia para Estudios Arqueológicos [Tesis de licenciatura, Universidad de Buenos Aires].

Samec, C. T. (2014). Ecología isotópica en la Puna Seca Argentina: un marco de referencia para el estudio de las estrategias de pastoreo en el pasado. Cuadernos del Instituto Nacional de Antropología y Pensamiento Latinoamericano, Series Especiales, 2(1), 61-85.

Samec, C. T. (2015). Estudio de las Relaciones entre los grupos Humanos y las Poblaciones de Camélidos en la puna seca Durante el Holoceno: una Aproximación a partir de los Isótopos Estables [Tesis doctoral, Universidad de Buenos Aires].

Samec, C. T., Morales, M. R. y Yacobaccio, H. D. (2014). Exploring human subsistence strategies and environmental change through stable isotopes in the Dry Puna Argentina. International Journal of Osteoarchaeology, 24, 134-148. https://doi.org/10.1002/oa.2332 
Samec, C. T. Yacobaccio, H. D. y Panarello, H. O. (2017). Carbon and nitrogen isotope composition of natural pastures in the dry Puna of Argentina: a baseline for the study of prehistoric herd management strategies. Archaeological and Anthropological Science, 9(2), 153163. https://doi.org/10.1007/s12520-015-0263-2

Samec, C. T., Yacobaccio, H. D. y Panarello, H. O. (2018). Stable isotope compositions of South American camelids in the Dry Puna of Argentina: A frame of reference for the study of prehistoric herding and hunting strategies. Journal of Archaeological Science: Reports, 18, 628-636. https://doi.org/10.1016/j. jasrep.2017.10.042

Srur, M. G., Izeta, A. D. y Scattolin, M. (2012). Alimentación de camélidos sudamericanos en los sitios formativos de Cardonal y Bordo Marcial (Catamarca, Argentina): Una aproximación isotópica. Archaeobios, 6(1), 5-18.

Stuiver, M., Reimer, P. J. y Reimer, R. W. (2020). CALIB 8.2 [WWW program]. http://calib.org (26 de diciembre 2020).

Szpak, P. (2013). Stable isotope ecology and humananimal interactions in Northern Peru. Electronic Thesis and Dissertation Repository. 1634. htts://ir.lib.uwo.ca/ etd/1634 (2 de julio 2019).

Szpak, P., White, C. D., Longstaffe, F. J., Millaire, J. F. y Vásquez Sánchez, V. F. (2013). Carbon and nitrogen isotopic survey of northern Peruvian plants: baselines for paleodietary and paleoecological studies. PLOS ONE, 8, e53763. https://doi.org/10.1371/journal. pone.0053763.t009

Thornton, E. K., Defrance, S. D., Krigbaum, J. y Williams, P. R. (2011). Isotopic evidence for Middle Horizon to 16th century camelid herding in the Osmore Valley, Peru. International Journal of Osteoarchaeology, 21, 544-567. https://doi.org/10.1002/oa.1157
Tieszen, L. L., Senyimba, M. M., Imbamba, S. K. y Troughton, J. H. (1979). The distribution of $C_{3}$ and $C_{4}$ grasses and carbon isotope discrimination along an altitudinal and moisture gradient in Kenya. Oecologia, 37, 337-350. https://doi.org/10.1007/BF00347910

Yacobaccio, H. D. (2001). La domesticación de camélidos en el Noroeste Argentino. En E. Berberián y A. E. Nielsen (Eds.), Historia Argentina Prehispánica, Tomo 1 (7-40). Brujas.

Yacobaccio, H. D. (2014). Pastoreo, movilidad y sequías. Cuadernos del Instituto Nacional de Antropología y Pensamiento Latinoamericano - Series Especiales, 2(1), 113-121.

Yacobaccio, H. D., Morales, M. R. y Samec, C. T. (2009). Towards an isotopic ecology of herbivory in the Puna ecosystem: new results and patterns on Lama glama. International Journal of Osteoarchaeology, 19, 144-155. https://doi.org/10.1002/oa.1050

Yacobaccio, H. D., Samec, C. T. y Catá, M. P. (2010). Isótopos estables y zooarqueología de camélidos en contextos pastoriles de la puna (Jujuy, Argentina). En M. A. Gutiérrez, M. De Nigris, P. M. Fernández, M. Giardina, A. Gil, A. Izeta, G. Neme y H. Yacobaccio (Eds.), Zooarqueología a Principios del siglo XXI. Aportes teóricos, metodológicos y casos de estudio (pp. 77-86). Ediciones del Espinillo.

Zavodny, E., McClure, S. B., Culleton, B. J., Podrug, E. y Kennett, D. J. (2015). Identifying Neolithic animal management practices in the Adriatic using stable isotopes. Documenta Praehistorica, XLII, 261-274. https://doi.org/10.4312/dp.42.18 